Document downloaded from:

http://hdl.handle.net/10251/103266

This paper must be cited as:

Jordi Burriel-Valencia; Puche-Panadero, R.; Martinez-Roman, J.; Sapena-Bañó, A.; PinedaSanchez, M. (2017). Short-Frequency Fourier Transform for Fault Diagnosis of Induction Machines Working in Transient Regime. IEEE Transactions on Instrumentation and Measurement. 66(3):432-440. doi:10.1109/TIM.2016.2647458

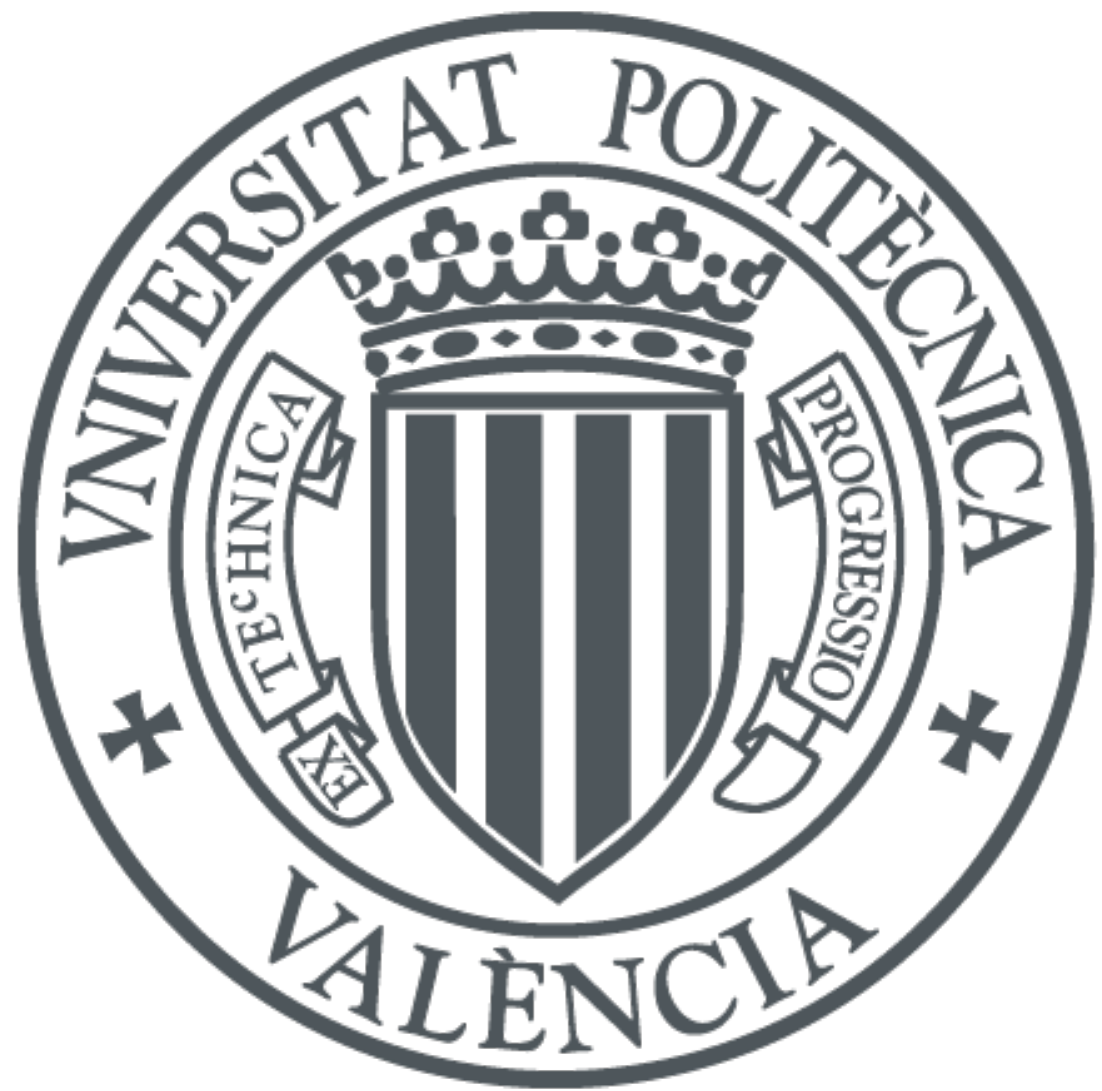

The final publication is available at

https://doi.org/10.1109/TIM.2016.2647458

Copyright Institute of Electrical and Electronics Engineers

Additional Information 


\title{
Short Frequency Fourier Transform for Fault Diagnosis of Induction Machines Working in Transient Regime
}

\author{
Jordi Burriel-Valencia, Ruben Puche-Panadero, Member, IEEE, Javier Martinez-Roman, Angel Sapena-Bano and \\ Manuel Pineda-Sanchez, Member, IEEE
}

\begin{abstract}
Transient-based methods for fault diagnosis of induction machines are attracting a rising interest, due to their reliability and ability to adapt to a wide range of induction machine (IM)'s working conditions. These methods compute the time-frequency (TF) distribution of the stator current, where the patterns of the related fault components can be detected. A significant amount of recent proposals in this field have focused on improving the resolution of the TF distributions, allowing a better discrimination and identification of fault harmonic components. Nevertheless, as the resolution improves, computational requirements (power computing and memory) greatly increases, restricting its implementation in low cost devices for performing on-line fault diagnosis. To address these drawbacks, in this paper the use of the short frequency Fourier transform (SFFT) for fault diagnosis of IMs working under transient regimes is proposed. The SFFT not only keeps the resolution of traditional techniques, such as the short time Fourier transform (STFT), but also achieves a drastic reduction of computation time and memory resources, making this proposal suitable for on-line diagnosis. This method is theoretically introduced and experimentally validated using a laboratory test bench.
\end{abstract}

Index Terms-Induction machines, fault diagnosis, short time Fourier transform, short frequency Fourier transform, timefrequency distributions, on-line diagnosis

\section{INTRODUCTION}

C AGE IMs are the keystone of electromechanical energy conversion in industrial installations, due to their reliability, robustness and low maintenance requirements. Despite these features, IMs are not free from having failures. Unexpected breakdowns can cause extremely heavy economic loses, ranging well beyond the self IM's cost. So, there is a growing interest in on-line condition monitoring methods [1], [2], whose main goal is to detect a fault at its early state without disturbing the IM's normal operation, allowing for taking an appropriate action plan.

Fault diagnosis of IMs via the current analysis is widely used because it is a non invasive method, has low hardware requirements (just one current must be sampled) and can identify a great variety of machine faults through the analysis

This work was supported by the Spanish "Ministerio de Economía y Competitividad" in the framework of the "Programa Estatal de Investigación, Desarrollo e Innovación Orientada a los Retos de la Sociedad" (project reference DPI2014-60881-R).

The authors are with the Department of Electrical Engineering, Universitat Politècnica de València, Valencia, Spain (e-mail: jorburva@ei.upv.es, rupucpa@die.upv.es,_jmroman@die.upv.es, ansaba2@upvnet.upv.es, mpineda@die.upv.es). of the current in the frequency domain, using the current spectrum for steady state regime [3], or the current TF distribution for transient regimes [4]. The frequencies of the fault harmonics that appear in the stator current have been theoretically introduced in the technical literature. For instance, a rotor broken bar fault induces or amplifies a set of harmonic components of frequencies $f_{b b}[5]$,

$$
f_{b b}=f_{\text {supply }}(1+2 k s) \quad k= \pm 1, \pm 2, \pm 3 \ldots,
$$

where $f_{\text {supply }}$ is the frequency of the power supply and $s$ is the slip. Other faults generate different, characteristic frequencies similar to (1).

From an industrial point of view, on-line fault diagnostic methods face practical difficulties. The spectral resolution must be high enough to detect the fault harmonics among a myriad of spectral lines that appear in the current spectrum [6], even in the case of a healthy machine [7]. Besides, the sampling rate must be high enough to reduce aliasing distortions. Modern hardware devices can operate at sampling rates well above $100 \mathrm{kHz}$ [8], so that the use of high sampling rates is common: $20 \mathrm{kHz}$ in [9], $50 \mathrm{kHz}$ in [10] and $100 \mathrm{kHz}$ in [11]. Additionally, the diagnostic procedure must be simple, compact and fast, to be executed on-line by embedded devices [6], [7], [12], or even by the same devices that control the IM, such as variable speed drives (VSDs). However, some of these requirements are conflicting ones. For example, to achieve a high spectral resolution, a long acquisition time is needed, which combined with high sampling rates implies that a huge amount of data must be stored and processed. This requires large size memory and high computing power, that is, costly devices and off-line diagnostic methods.

These drawbacks have a major impact in transientbased fault diagnosis methods, which require advanced and complex signal processing tools such as the STFT [13], [14], the wavelet transform [15], [16], the empirical mode decomposition [17], [18], the Wigner Ville distribution (WVD) [19], among many others. Wavelet-based transforms require a proper mother wavelet choice and a precise adjustment of the bands of decomposition to perform fault diagnosis. Quadratic-based TF transforms, such as the WVD, generate cross-terms effects that can difficult the evaluation of the spectrogram, and their minimization has been widely discussed in the technical literature [20]-[22]. On the contrary, these problems do not appear if the linear-based TF transforms, such as the STFT, are used, so the STFT has been selected 
in this paper to carry out the fault diagnosis in transient regime. All these TF transforms generate three dimensional (time-frequency-amplitude) representations, instead of the two dimensional representation (frequency-amplitude) used in steady state, which increases substantially the computing power and memory requirements of any diagnostic system. Hence the complexity of these signal processing-based condition monitoring techniques have limited their use in realtime applications [23], specially in field programmable gate array (FPGA) [13], [24], digital signal processor (DSP) [25] or even in general-purpose graphics processing units (GPGPUs) [23].

To sum up, high sampling rates combined with long acquisition times generate a large amount of data which must be stored and processed, specially when using TF transforms. But there is an important fact in the field of the fault diagnosis of IMs that can alleviate these problems: the relevant information about the main fault harmonics is limited to a very small range of frequencies $([0,100] \mathrm{Hz}$ in case of rotor broken bars or mixed eccentricity, for example). So, filtering the current signal in the frequency domain only in this reduced range of frequencies, instead of filtering the current signal in the time domain and discarding afterwards all the frequency data outside the band of interest, as is usually done, can reduce drastically the required resources. This approach has been recently proposed in the discrete case [4] using the fast Fourier transform (FFT), and is extended in this paper to the continuous domain, using the SFFT.

The structure of this paper is as follows: the theoretical basis of the STFT and the SFFT are presented in Section II. The election of the proposed method is justified in Section III, and illustrated in Section IV using a fault component extracted from the current of a simulated machine with a rotor broken bar. In Section $\mathrm{V}$ the experimental validation is carried out using a laboratory test bench. In both sections, (Section IV and Section V) there is a comparative analysis in terms of computation power and memory requirement between the traditional method, based on the STFT, and the proposed method, based on the SFFT. Finally, in Section VI the main conclusions of this work are presented.

\section{TheOrETICAL BACKGROUND}

This section compares theoretically the continuous transform used traditionally for fault diagnosis of IMs, the STFT (which uses filters in the time domain), and the proposed technique based on the use of the SFFT (which uses filters in the frequency domain).

\section{A. Filtering in the time-domain: the short time Fourier transform (STFT)}

The STFT is a well-known technique used for studying non-stationary signals in industrial applications. The STFT of currents and vibrations has been extensively used for detecting, in transient regime, different types of IM's faults such as rotor broken bars or damaged bearings [26], among others.

Given a time-domain current signal $i(\tau)$, the STFT uses a window function $h(\tau)$ (assuming unitary amplitude) centred at time $t$ to obtain a modified signal $i_{t}(\tau)$, which emphasizes the original signal $i(\tau)$ at that time $t$, and suppresses the signal at other times. In other words, the non-stationary signal can be considered as locally stationary, and then can be transformed into the time-frequency domain [27],

$$
i_{t}(\tau)=i(\tau) h(\tau-t) .
$$

Hence, the modified signal is a function of two times, $t$ and $\tau$, as:

$$
i_{t}(\tau) \simeq\left\{\begin{array}{l}
i(\tau) \text { if } \tau \text { is near to } t \\
0 \text { if } \tau \text { is far away from } t .
\end{array}\right.
$$

The Fourier transform of the windowed signal reflects the frequency distribution around time $t$,

$$
I_{t}(\omega)=\frac{1}{\sqrt{2 \pi}} \int e^{-j \omega \tau} i(\tau) h(\tau-t) \mathrm{d} t
$$

and the energy density spectrum at time $t$ is

$$
P_{S P}(t, \omega)=\left|I_{t}(\omega)\right|^{2}=\left|\frac{1}{\sqrt{2 \pi}} \int e^{-j \omega \tau} i(\tau) h(\tau-t) \mathrm{d} \tau\right|^{2} .
$$

For each different time the STFT generates a different spectrum, and the totality of these spectra is the spectrogram.

\section{B. Filtering in the frequency-domain: the short frequency Fourier transform (SFFT)}

As the STFT emphasizes the study of frequency properties at specific time $t$, the SFFT allows to study the time properties at a particular frequency $\omega$, being the relation of the current signal in time $i(t)$ with the current signal in frequency $I(\omega)$ the Fourier transform

$$
I(\omega)=\int i(t) e^{-j \omega t} \mathrm{~d} t
$$

Similarly to the STFT, the SFFT uses a window $H\left(\omega^{\prime}\right)$ centred at frequency $\omega$ to obtain a modified current signal $I_{\omega}$, which emphasizes the original signal $I\left(\omega^{\prime}\right)$ at that frequency $\omega$, and suppresses the signal at other frequencies,

$$
I_{\omega}\left(\omega^{\prime}\right)=I\left(\omega^{\prime}\right) H\left(\omega^{\prime}-\omega\right) .
$$

Hence, the modified signal is a function of two frequencies, $\omega$ and $\omega^{\prime}$ :

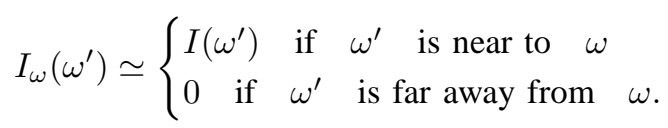

The inverse Fourier transform of the windowed signal reflects the time distribution around the frequency $\omega$

$$
i_{\omega}(t)=\frac{1}{\sqrt{2 \pi}} \int e^{j \omega^{\prime} t} I\left(\omega^{\prime}\right) H\left(\omega^{\prime}-\omega\right) \mathrm{d} \omega^{\prime},
$$

and the energy density spectrum at frequency $\omega$ is

$$
P_{S P}(t, \omega)=\left|i_{\omega}(t)\right|^{2}=\left|\frac{1}{\sqrt{2 \pi}} \int e^{j \omega^{\prime} t} I\left(\omega^{\prime}\right) H\left(\omega^{\prime}-\omega\right) \mathrm{d} \omega^{\prime}\right|^{2}
$$

Applying (6) to (9) [28]

$$
I_{t}(\omega)=e^{-j \omega t} i_{\omega}(t),
$$


Short Time Fourier Transform (STFT)

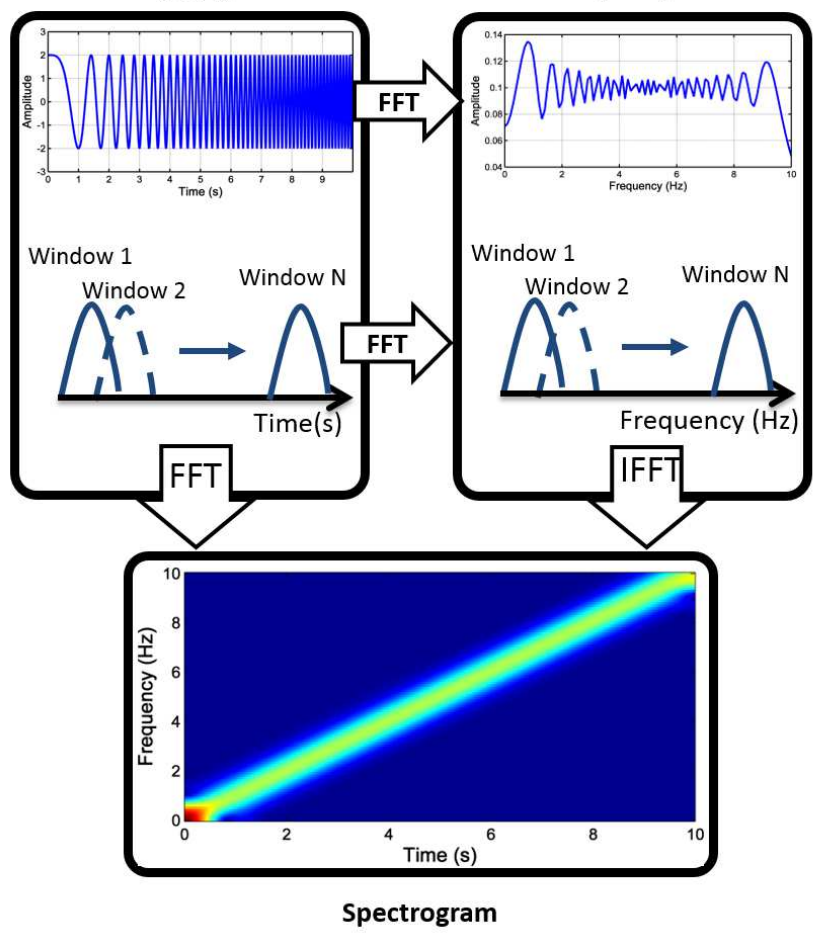

Fig. 1. Steps to obtain the spectrogram (bottom) of a synthetic chirp signal $y(t)=\cos \left(0.8 \pi t^{2}\right)$ via the STFT (top, left) or via the SFFT (top, right). The results are exactly the same.

so the STFT and the SFFT obtain the same result except for the phase factor $e^{-j \omega t}$. Since the energy density spectrum is an absolute square, the phase factor $e^{-j \omega t}$ does not affect it and either the STFT or the SFFT can be used to generate the joint distribution, $P_{S P}(t, \omega),(5)$ and (10)

$$
P_{S P}(t, \omega)=\left|I_{t}(\omega)\right|^{2}=\left|i_{\omega}(t)\right|^{2},
$$

as depicted in Fig. 1. This figure shows the spectrogram of a synthetic chirp signal $y(t)=\cos \left(0.8 \pi t^{2}\right)$ obtained using either the STFT or the SFFT. The results are exactly the same, as expected.

\section{Proposed Method: Fault Diagnosis of Rotating ELECTRICAL MACHINES VIA THE SFFT}

As can be deduced from Section II-A and Section II-B, the same spectrogram can be obtained via the STFT and the SFFT. So, why is interesting the use of the SFFT as a method for fault diagnosis? As can be seen in Fig. 1, both methods use a window which is moved either along the time axis (STFT) or along the frequency axis (SFFT) to obtain the spectrogram of the current signal. Nevertheless, if the STFT is used, the window must be shifted along the full-length of the signal, which has been sampled at a frequency $F_{\text {sampling }}$. Besides, although in any step (window translation) a spectrum from $\left[-F_{\text {sampling }} / 2, F_{\text {sampling }} / 2\right]$ is obtained, for fault diagnosis the related main fault components appear in a very limited frequency band (for example $[0,50] \mathrm{Hz}$ for components due to rotor broken bar or mixed eccentricity during the startup transient of an IM). Hence, in any step of the STFT, a large amount of data, which is not relevant for fault diagnosis, must be discarded, because the spectrum stores frequencies far beyond the needs of diagnostic data. This drawback is more pronounced with the higher sampling frequencies currently used for fault diagnosis.

These problems can be greatly reduced with the use of the SFFT. The translation of the window in the frequency domain can be limited to the band covered by the expected fault frequencies. For example, in case of a rotor broken bar, the left sideband harmonic (LSH), $k=-1$ in (1), is one of the most widely studied in fault diagnosis of IMs working in transient regime. Its frequency during the start-up of the machine varies from the frequency of the power supply $f_{\text {supply }}$ (usually $50-60 \mathrm{~Hz}$ ) to $0 \mathrm{~Hz}$ and then grows up to its value in steady state (close to $f_{\text {supply }}$ ). In this case, the SFFT can be limited to study the frequency band $\left[0, f_{\text {supply }}\right]$, improving the diagnostic procedure in two ways. First, this reduces the number of translations of the window and, therefore, the computational resources and the time to obtain the spectrogram. On the other hand, in any step the time properties at a particular frequency of interest are obtained, so the full data obtained is useful for fault diagnosis. This procedure is valid for any type of fault. Therefore the current spectrogram obtained using the SFFT in a limited frequency band contains the same information, in terms of fault diagnosis, as the one obtained by the STFT, but using far less resources. This allows for computing spectrograms on-line using low cost digital signal devices such as DSPs or FPGAs.

\section{CASE of Study: LSH Due to A Rotor Broken Bar DURING THE START-UP OF A INDUCTION MACHINE}

Prior to the experimental validation, the proposed method and its advantages are illustrated in this section using the evolution of the LSH due to a rotor broken bar during the start-up of an IM. This harmonic component was extracted in [29] from a simulated machine (whose characteristics are presented in Appendix A), considering only the fundamental space harmonics of its windings, sampled during $T=2 \mathrm{~s}$ at $F_{\text {sampling }}=5 \mathrm{kHz}$, giving a total of $N=10^{4}$ samples. Basically, the LSH is a sinusoidal wave whose amplitude and frequency vary continuously depending on the slip $s$. The amplitude pattern of the LSH is shown in Fig. 2. First, the amplitude decreases until it becomes null $(s=0.5, t=0.92 \mathrm{~s}$ ). From this point, the amplitude increases until it reaches a maximum. Finally, the amplitude decreases toward its steady state value.

Similarly, the frequency of the LSH (Fig. 3) decreases from $50 \mathrm{~Hz}(s=1)$ until it becomes null $(s=0.5, t=0.92 \mathrm{~s})$. In the second half of the transient $(s>0.5, t>0.92 \mathrm{~s})$ the frequency increases, keeping a constant value when the steady state is reached.

To obtain the spectrogram of the LSH a Gaussian window $h(t)$ has been used to compute both the STFT and the SFFT

$$
h(t)=(\alpha / \pi)^{1 / 4} e^{-\alpha t^{2} / 2},
$$



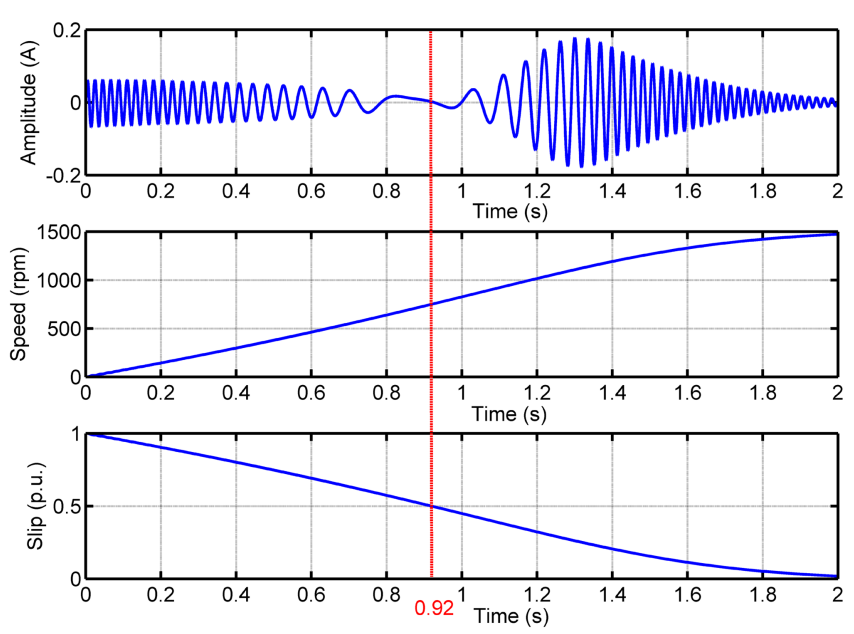

Fig. 2. Amplitude of the LSH (top), motor speed (middle), and motor slip (bottom) during the start-up transient of a simulated induction machine with a rotor broken bar. The vertical line corresponds to the time when the slip $s=0.5$ is reached.

\section{Frequency of the LSH}

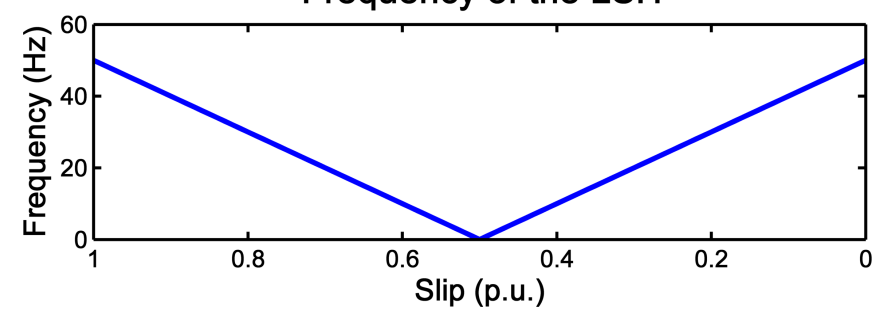

Fig. 3. Evolution of the frequency of the LSH as a function of the rotor slip.

, where $\alpha$ is selected so that the Heisenberg box of the window achieves the maximum overlap with the trajectory of the fault harmonic component in the spectrogram, as in [29]. The optimum value of $\alpha$ for this fault harmonic component is $\alpha^{\text {opt }}=341.5$. This function is represented in the time and frequency domains in Fig. 4.
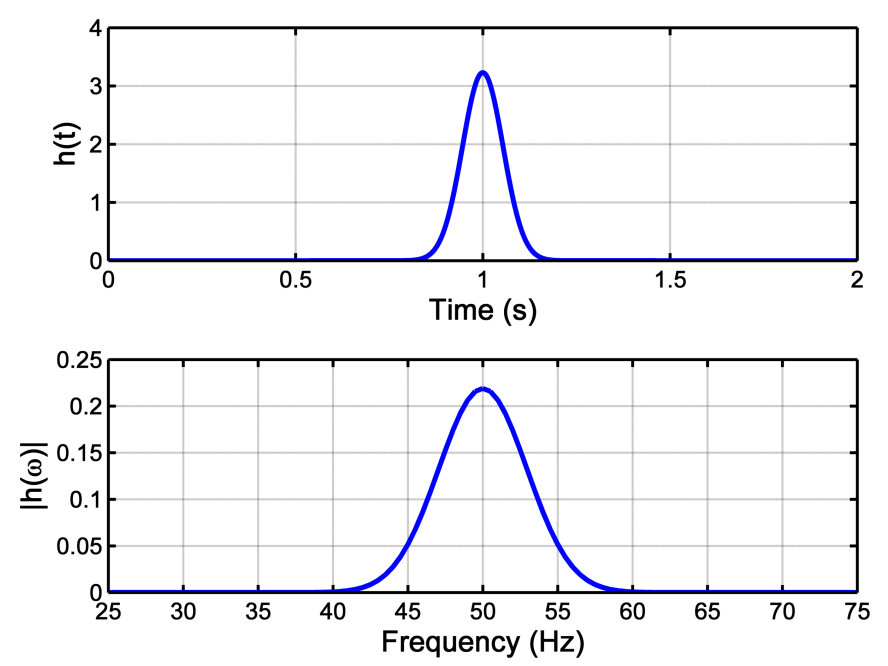

Fig. 4. Gaussian window optimized for representing the LSH in the time domain (top) and frequency domain (bottom).

This window is applied to the LSH (Fig. 2, top) to obtain the spectrogram using both the STFT and the SFFT. As can be seen in Fig. 3, the fault harmonic component evolves in the the frequency band $[0,50] \mathrm{Hz}$. Hence, the frequency band where the window is moved using the proposed method, the SFFT, has been limited to $[0,60] \mathrm{Hz}$.

Fig. 5 shows the time-frequency-amplitude pattern generated by the LSH using the STFT, and with the proposed method, using the SFFT. As indicated in section II, the spectrogram are identical, regardless the method used. If the STFT is used, in any time step a window is applied to the signal, the spectrum of the windowed signal is obtained, and a huge amount of data irrelevant for fault diagnosis is removed to keep just the useful data (in the frequency band $[0,60] \mathrm{Hz}$ ) which is stored in each step of the transform. In contrast, if the SFFT is used, the irrelevant data outside the $[0,60] \mathrm{Hz}$ band is removed only in the first step. Moreover the number of steps to compute the time frequency distribution is lower if the SFFT, $N_{F}$, is used instead of the STFT, $N_{T}$, keeping the same results. In fact, not only the number of steps is reduced, but also the power computing, the computation time, and the memory requirements are downsized.

Considering the total number of bins of the full-length signal of Fig. 2, $M$, as

$$
M=F_{\text {sampling }} \cdot T=5 \cdot 10^{3} \cdot 2=10^{4} \text { bins, }
$$

when moving the window every single bin, the number of window translations, or steps, in case of the STFT is $N=M=10^{4}$ bins. On the contrary, if the SFFT is used, the window is just moved between $[0,60] \mathrm{Hz}$ in the frequency axis. Considering $K_{f}$ the bin number corresponding to a given frequency $f$

$$
K_{f}=f \frac{N}{F_{\text {sampling }}} \rightarrow\left\{\begin{array}{l}
K_{f}=0 \text { if } f=0 \\
K_{f}=120 \text { if } f=60,
\end{array}\right.
$$

then the proposed procedure involves moving the window between the bins range $[0,120]$, and therefore just $N_{F}=121$ steps are needed to obtain the spectrogram in the frequency band where the fault component is expected to appear. This diagnostic spectrogram has the same information about the main fault components as the one obtained with the STFT. However, there is a substantial difference regarding the computational cost and memory requirements. Table I shows a comparative analysis between the STFT and the SFFT in terms of number of steps, computation time and data processed. These results have been obtained using a personal computer whose main characteristics are exposed in the Appendix C. As can be seen in Table I, the requirements to obtain the diagnostic spectrogram have been drastically reduced if the proposed method, based on the SFFT, is used, instead of using the STFT. In fact, the use of the SFFT reduces in almost $99 \%$ the resources required to perform the diagnosis.

\section{EXPERIMENTAL VALIDATION}

The experimental validation has been performed under laboratory conditions, using a commercial motor, whose main characteristics are given in the Appendix B, with an artificially 


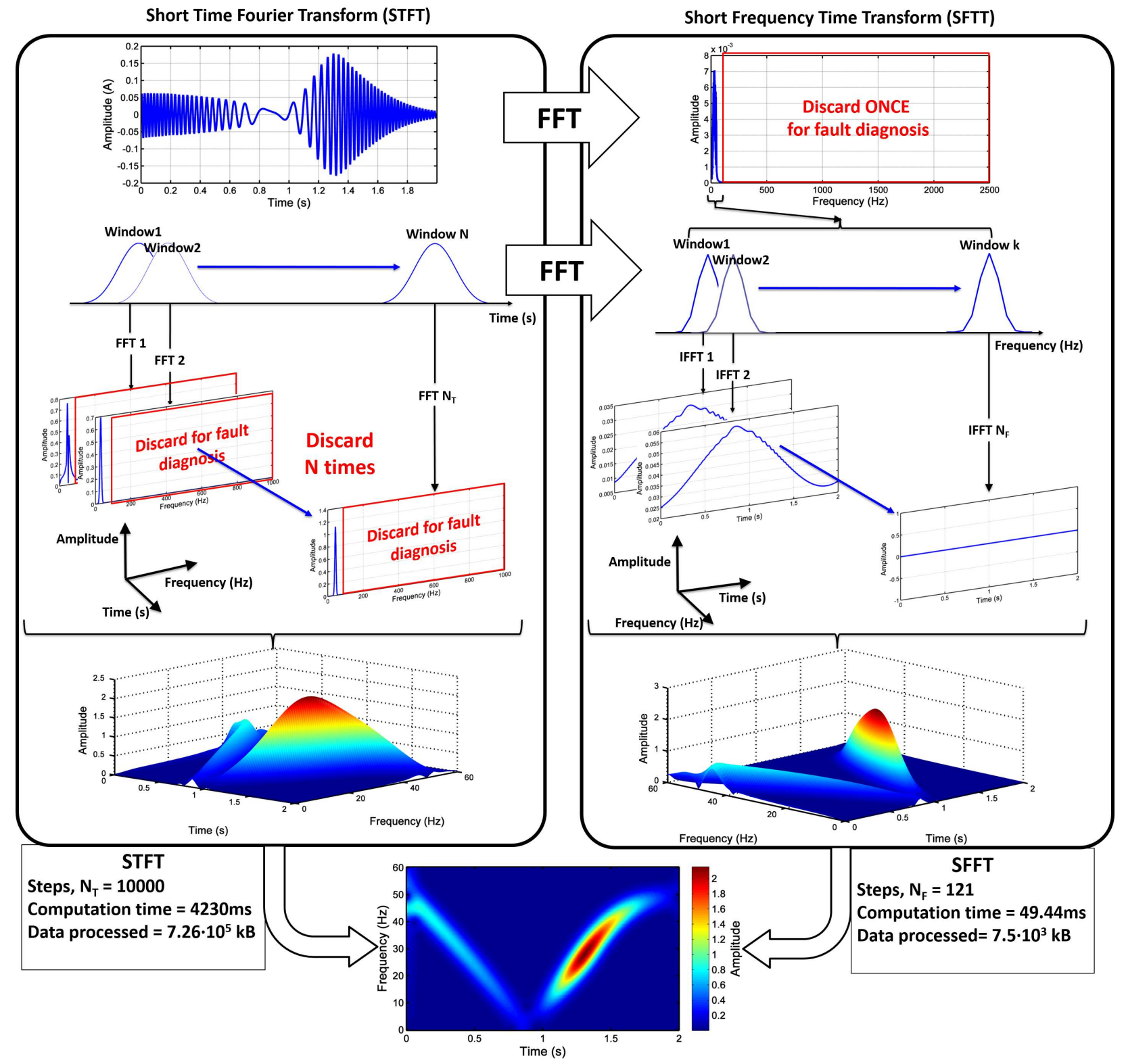

Time-Frequency-Amplitude distribution

Fig. 5. Steps to obtain the diagnostic spectrogram of the LSH (case of study section IV) using the STFT and the proposed method based on the use of the SFFT. If the STFT is used, at each step the full spectrum of the windowed signal is obtained, and a huge amount of data, irrelevant for fault diagnosis, is removed. In contrast, if the SFFT is applied, the irrelevant data are removed only at the first step. Besides, the number of the window displacements is far smaller. Hence, the proposed method reduces drastically the resources required.

TABLE I

COMPARATIVE ANALYSis OF THE Diagnostic Computational Cost USING THE STFT AND THE PROPOSED METHOD BASED ON SFFT

\begin{tabular}{cccc}
\hline \hline & \multicolumn{2}{c}{ Comparative analysis } & Reduction \\
& (A) STFT & (B) SFFT & (A-B)/A (\%) \\
\hline Steps & 10000 & 121 & 98.79 \\
Computation time (ms) & 4230 & 49.44 & 98.83 \\
Data processed $(\mathrm{kB})$ & 726232 & 7543 & 98.96 \\
\hline \hline
\end{tabular}

rotor broken bar (Fig. 6), mounted in the test bench depicted in Fig. 7.

The IM under test is fed through a VSD model ABB ACS800-01-0005-3 (Fig. 7, left). As a mechanical load a permanent magnet synchronous machine (PMSM) has been used (Fig. 7, bottom). It is fed through a servo drive model ABB ACSM1-04AS-024A-4 (Fig. 7, right). The control method of the VSD has been established in open-loop constant voltage per frequency $(\mathrm{CV} / \mathrm{F})$. Three tests, whose main characteristics are shown in Table II, have been performed. During the tests the output frequency of the VSD has been changed linearly between two levels $f_{\min }$ and $f_{\max }$ each $t_{\text {ramp }}$ seconds. The value of $\alpha$ to build the window (13) has 
been computed according to [29] as

$$
\alpha=\frac{2 \pi\left(f_{\max }-f_{\min }\right)}{t_{\text {ramp }}} .
$$

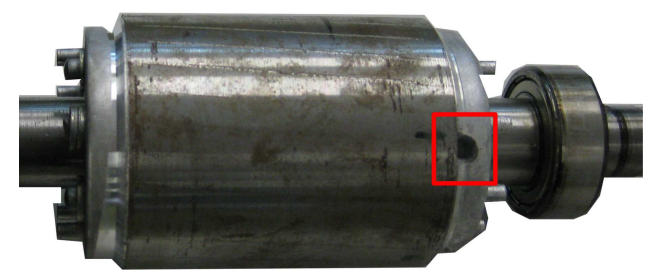

Fig. 6. Rotor with an artificially broken bar used in the experimental tests.

TABLE II

LABORATORY TESTS PERFORMED

\begin{tabular}{|c|c|c|c|}
\hline & \multicolumn{3}{|c|}{ Properties of the tests } \\
\hline & case 1 & case 2 & case 3 \\
\hline Time test, $T$ (s) & 10 & 10 & 10 \\
\hline Sampling frequency, $F_{\text {sampling }}(\mathrm{kHz})$ & 5 & 5 & 5 \\
\hline$N=F \cdot T$ (bins) & $5 \cdot 10^{4}$ & $5 \cdot 10^{4}$ & $5 \cdot 10^{4}$ \\
\hline Load $\%$ & 70 & 50 & 100 \\
\hline$f_{\min }(\mathrm{Hz})$ & 20 & 45 & 40 \\
\hline$f_{\max }(\mathrm{Hz})$ & 25 & 50 & 50 \\
\hline$t_{\text {ramp }}(\mathrm{s})$ & 10 & 10 & 10 \\
\hline eq. (16) & $\pi$ & $\pi$ & $2 \pi$ \\
\hline
\end{tabular}

The level of load of each test has been adjusted using the torque control of the servo drive which supplies the PMSM. This level has been established as a percentage of the rated load of the tested IM. Additionally, the speed has been sampled through the resolver sensor coupled to the PMSM rotor. The tests have been performed during $10 \mathrm{~s}$ at a sampling frequency of $5 \mathrm{kHz}$, as shown in Table II. Both signals allow to obtain the spectrogram of the current and to compute the trajectories that the fault components follow in the time-frequency plane.

TABLE III

COMPARISON OF THE STEPS NEEDED TO OBTAIN THE Diagnostic SPECTROGRAM USING THE STFT AND THE SFFT

\begin{tabular}{cccc}
\hline \hline & \multicolumn{2}{c}{ Number of steps } & Reduction \\
& (A) STFT & (B) SFFT & (A-B)/A (\%) \\
\hline Case 1 & 50000 & 351 & 99.30 \\
Case 2 & 50000 & 251 & 99.50 \\
Case 3 & 50000 & 341 & 99.32 \\
\hline \hline
\end{tabular}

The results of applying both the STFT and the SFFT to these tests are shown in Fig. 8. The diagnostic spectrograms are the same regardless the method used. The four black lines in each spectrogram are the theoretical patterns of the fault components corresponding to the orders $k= \pm 1, \pm 2$ in (1) for each test. They appear in the spectrogram overlapping harmonic components, so the presence of faults in the machine is confirmed.

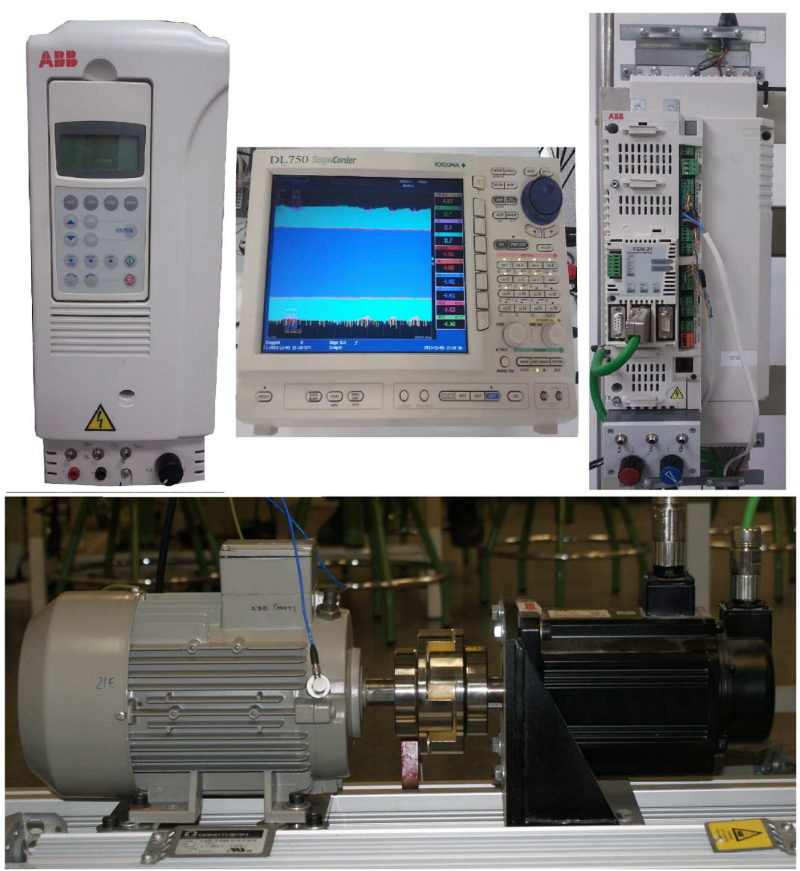

Fig. 7. Laboratory test bench. Top, ABB VSD (left), digital oscilloscope (center) and servo drive (right). Bottom, cage induction machine coupled to a permanent magnet synchronous machine.

TABLE IV

COMPARISON OF THE COMPUTATION TIME NEEDED TO OBTAIN THE Diagnostic SPECTROGRAM USING THE STFT AND THE SFFT

\begin{tabular}{cccc}
\hline \hline & \multicolumn{2}{c}{ Computation time (s) } & Reduction \\
& (A) STFT & (B) SFFT & (A-B)/A $(\%)$ \\
\hline Case 1 & 121.05 & 1.13 & 99.07 \\
Case 2 & 118.60 & 0.79 & 99.33 \\
Case 3 & 117.37 & 1.10 & 99.06 \\
\hline \hline
\end{tabular}

Moreover, a comparative analysis between the application of the STFT and the SFFT has been performed using the three test cases of Table II. This comparison has been developed using the computer whose main features are shown in Appendix C. Tables III and IV show reduction rates of several orders of magnitude both in the number of steps and in the computing time using the SFFT, where the window is moved in the frequency domain, but just in the frequency bands of interest: $[0,35] \mathrm{Hz}$ case $1,[35,60] \mathrm{Hz}$ case 2 and $[28,62] \mathrm{Hz}$ case 3 . Besides, as can be seen in Table $\mathrm{V}$, a huge reduction on the amount of memory required to compute the spectrogram has been achieved. Despite this reduction on computation resources, both methods achieve the same diagnostic results, making the proposed method, based on the use of the SFFT, suitable to be implemented in low costs devices.

\section{CONCLUSION}

In this paper a novel method for fault diagnosis of IM has been introduced. The proposed method reduces considerably the computational cost and the amount of memory required to obtain the diagnostic spectrogram of the stator current, while keeping the results obtained using traditional diagnostic 

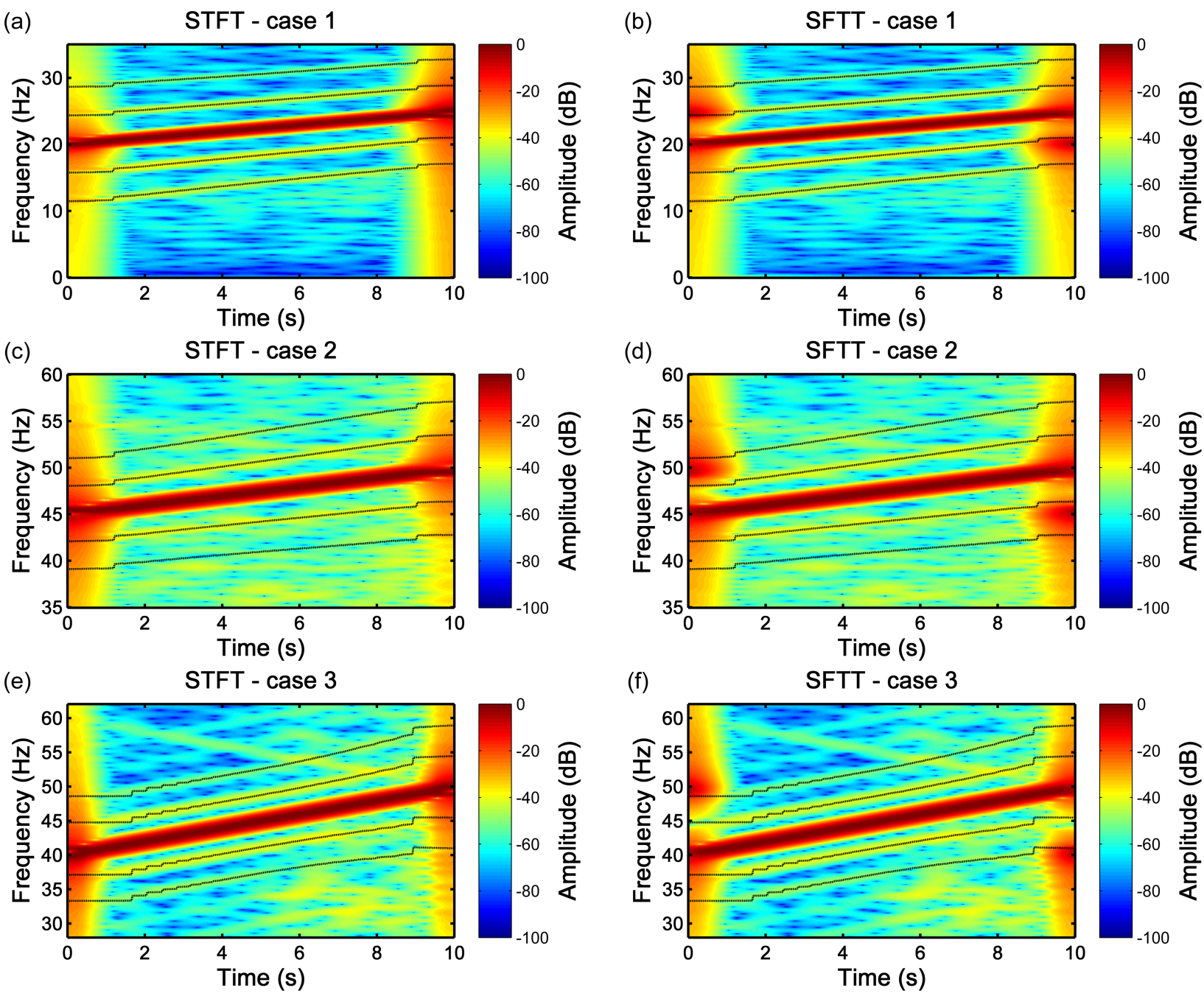

Fig. 8. Diagnostic spectrogram generated by the currents of the three tests using the STFT: (a) case 1, (c) case 2, (e) case 3. And using the proposed method based on the SFFT: (b) case 1, (d) case 2 and (f) case 3. In black, four lines are drawn which are the theoretical pattern of the harmonic fault components of orders $k= \pm 1, \pm 2$ obtained from (1)

TABLE V

Comparison of the Amount of Memory NeEded to Store the DiAGNOSTIC SPECTROGRAM USING THE STFT AND THE SFFT

\begin{tabular}{lccc}
\hline \hline & \multicolumn{2}{c}{ Data processed (kB) } & Reduction \\
& (A) STFT & (B) SFFT & $($ A-B)/A (\%) \\
\hline Case 1 & $1.76 \cdot 10^{7}$ & 124013 & 99.3 \\
Case 2 & $1.75 \cdot 10^{7}$ & 88214 & 99.50 \\
Case 3 & $1.78 \cdot 10^{7}$ & 121867 & 99.32 \\
\hline \hline
\end{tabular}

method based on the STFT. The proposed method, based on the SFFT, relies on filtering in the frequency domain, but just in the frequency band of interest for fault diagnosis, instead of filtering in the time-domaing along the full-length of the signal, as using the STFT. This results in a huge reduction of computational resources, more than $99 \%$ of reduction in number of steps, computing time and memory resources, allowing its implementation using low cost devices. The proposed method has been theoretically introduced and illustrated using the LSH signal of a simulated machine.
Finally, it has been validated using three tests performed at different levels of load and speed using a commercial IM with a rotor broken bar.

\section{APPENDIX A \\ Simulated MACHINE}

Three-phase induction machine. Rated characteristics: $P=$ $1.1 \mathrm{~kW}, f=50 \mathrm{~Hz}, U=230 / 400 \mathrm{~V}, I=2.7 / 4.6 \mathrm{~A}, n=$ 1410 r.p.m., $\cos \varphi=0.8$. Load inertia $0.25 \mathrm{~kg} \cdot \mathrm{m}^{2}$.

\section{APPENDIX B}

\section{Cage Induction Machine Mounted in the} LABORATORY TEST BENCH

Three-phase induction machine, star connection. Rated characteristics: $P=1.5 \mathrm{~kW}, f=50 \mathrm{~Hz}, U=400 \mathrm{~V}$, $I=3.25 \mathrm{~A}, n=2860 \mathrm{rpm}$, and $\cos \varphi=0.85$.

\section{APPENDIX C}

\section{COMPUTER FEATURES}

CPU: Intel Core i7-2600K CPU @ 3.40 GHZ RAM memory: $16 \mathrm{~GB}$, Matlab Version: 8.0.0.783 (R2012b). 


\section{REFERENCES}

[1] L. Medina, R. de Jesus Romero-Troncoso, E. Cabal-Yepez, J. de Jesus Rangel-Magdaleno, and J. Millan-Almaraz, "FPGA-Based MultipleChannel Vibration Analyzer for Industrial Applications in Induction Motor Failure Detection," IEEE Trans. Instrum. Meas., vol. 59, no. 1, pp. 63-72, Jan 2010.

[2] S. Yin, S. Ding, X. Xie, and H. Luo, "A Review on Basic DataDriven Approaches for Industrial Process Monitoring," IEEE Trans. Ind. Electron., vol. 61, no. 11, pp. 6418-6428, Nov 2014.

[3] D. Z. Li, W. Wang, and F. Ismail, "An Enhanced Bispectrum Technique With Auxiliary Frequency Injection for Induction Motor Health Condition Monitoring," IEEE Trans. Instrum. Meas., vol. 64, no. 10 , pp. 2679-2687, Oct 2015.

[4] A. Sapena-Bano, M. Pineda-Sanchez, R. Puche-Panadero, J. MartinezRoman, and D. Matic, "Fault Diagnosis of Rotating Electrical Machines in Transient Regime Using a Single Stator Current's FFT," IEEE Trans. Instrum. and Meas., vol. 64, no. 11, pp. 3137-3146, Nov 2015.

[5] R. Romero-Troncoso, A. Garcia-Perez, D. Morinigo-Sotelo, O. DuquePerez, R. Osornio-Rios, and M. Ibarra-Manzano, "Rotor unbalance and broken rotor bar detection in inverter-fed induction motors at start-up and steady-state regimes by high-resolution spectral analysis," Electric Power Systems Research, vol. 133, pp. 142 - 148, April 2016.

[6] B. Akin, S. Choi, U. Orguner, and H. Toliyat, "A Simple Real-Time Fault Signature Monitoring Tool for Motor-Drive-Embedded Fault Diagnosis Systems," IEEE Trans. Ind. Electron.,, vol. 58, no. 5, pp. 1990-2001, May 2011.

[7] G. Joksimovic, J. Riger, T. Wolbank, N. Peric, and M. Vasak, "Stator-Current Spectrum Signature of Healthy Cage Rotor Induction Machines," IEEE Trans. Ind. Electron., vol. 60, no. 9, pp. 4025-4033, Sept 2013.

[8] I. P. Georgakopoulos, E. D. Mitronikas, and A. N. Safacas, "Detection of Induction Motor Faults in Inverter Drives Using Inverter Input Current Analysis," IEEE Trans. Indus. Electron., vol. 58, no. 9, pp. 4365-4373, Sept 2011.

[9] S. Toscani, M. Faifer, M. Rossi, L. Cristaldi, and M. Lazzaroni, "Effects of the Speed Loop on the Diagnosis of Rotor Faults in Induction Machines," IEEE Trans. Instrum. Meas., vol. 61, no. 10, pp. 2713-2722, Oct 2012.

[10] K. Shahzad, P. Cheng, and B. Oelmann, "Architecture Exploration for a High-Performance and Low-Power Wireless Vibration Analyzer," IEEE Sens. J., vol. 13, no. 2, pp. 670-682, Feb 2013.

[11] A. Sapena-Bano, M. Pineda-Sanchez, R. Puche-Panadero, J. Perez-Cruz, J. Roger-Folch, M. Riera-Guasp, and J. Martinez-Roman, "Harmonic Order Tracking Analysis: A Novel Method for Fault Diagnosis in Induction Machines," IEEE Trans. Energy Convers., vol. 30, no. 3, pp. 833-841, Sept 2015.

[12] A. Lebaroud and A. Medoued, "Online computational tools dedicated to the detection of induction machine faults," International Journal of Electrical Power \& Energy Systems, vol. 44, no. 1, pp. 752 - 757, Jan 2013.

[13] E. Cabal-Yepez, A. Garcia-Ramirez, R. Romero-Troncoso, A. GarciaPerez, and R. Osornio-Rios, "Reconfigurable Monitoring System for Time-Frequency Analysis on Industrial Equipment Through STFT and DWT," IEEE Trans. Ind. Inf., vol. 9, no. 2, pp. 760-771, May 2013.

[14] S. Nandi, T. Ilamparithi, S. B. Lee, and D. Hyun, "Detection of Eccentricity Faults in Induction Machines Based on Nameplate Parameters," IEEE Trans. Ind. Electron., vol. 58, no. 5, pp. 1673-1683, May 2011.

[15] R. Yan, R. X. Gao, and X. Chen, "Wavelets for fault diagnosis of rotary machines: a review with applications," Signal Processing, vol. 96, pp. 1-15, March 2014

[16] J. Chen, J. Pan, Z. Li, Y. Zi, and X. Chen, "Generator bearing fault diagnosis for wind turbine via empirical wavelet transform using measured vibration signals," Renewable Energy, vol. 89, pp. 80 - 92, April 2016.

[17] R. Valles-Novo, J. de Jesus Rangel-Magdaleno, J. Ramirez-Cortes, H. Peregrina-Barreto, and R. Morales-Caporal, "Empirical Mode Decomposition Analysis for Broken-Bar Detection on Squirrel Cage Induction Motors," IEEE Trans. Instrum. Meas., vol. 64, no. 5, pp. 11181128, May 2015.

[18] F. Batista, P. Lamim Filho, R. Pederiva, and V. Silva, "An Empirical Demodulation for Electrical Fault Detection in Induction Motors," IEEE Trans. Instrum. Meas., vol. 65, no. 3, pp. 559-569, March 2016.

[19] V. Climente-Alarcon, J. Antonino-Daviu, A. Haavisto, and A. Arkkio, "Diagnosis of Induction Motors Under Varying Speed Operation by
Principal Slot Harmonic Tracking," IEEE Trans. Ind. Appl., vol. 51, no. 5, pp. 3591-3599, Sept 2015 .

[20] R. B. Pachori and A. Nishad, "Cross-terms reduction in the Wigner-Ville distribution using tunable-Q wavelet transform ," Signal Processing, vol. 120, pp. 288 - 304, March 2016.

[21] H. Ren, A. Ren, and Z. Li, "A new strategy for the suppression of crossterms in pseudo Wigner-Ville distribution," Signal, Image and Video Processing, vol. 10, no. 1, pp. 139-144, Jan 2016.

[22] M. Damir, S. Victor, and C. Zlatan, "Optimizing the Reference Signal in the Cross Wigner-ville Distribution Based Instantaneous Frequency Estimation Method ," Procedia Engineering, vol. 100, pp. 1657 - 1664, 2015.

[23] M. Kang, J. Kim, and J.-M. Kim, "High-Performance and EnergyEfficient Fault Diagnosis Using Effective Envelope Analysis and Denoising on a General-Purpose Graphics Processing Unit," IEEE Trans. Power Electron., vol. 30, no. 5, pp. 2763-2776, May 2015.

[24] J. de Jesus Rangel-Magdaleno, H. Peregrina-Barreto, J. Ramirez-Cortes, P. Gomez-Gil, and R. Morales-Caporal, "FPGA-Based Broken Bars Detection on Induction Motors Under Different Load Using Motor Current Signature Analysis and Mathematical Morphology," IEEE Trans. Instrum. Meas., vol. 63, no. 5, pp. 1032-1040, May 2014.

[25] S. Choi, B. Akin, M. Rahimian, and H. Toliyat, "Implementation of a Fault-Diagnosis Algorithm for Induction Machines Based on Advanced Digital-Signal-Processing Techniques," IEEE Trans. Indus. Electron., vol. 58, no. 3, pp. 937-948, March 2011.

[26] H. Gao, L. Liang, X. Chen, and G. Xu, "Feature extraction and recognition for rolling element bearing fault utilizing short-time Fourier transform and non-negative matrix factorization," Chinese Journal of Mechanical Engineering, vol. 28, no. 1, pp. 96-105, Dec 2015.

[27] L. Cohen, "Time-frequency distributions-a review," Proc.IEEE, vol. 77, no. 7, pp. 941-981, July 1989.

[28] M. Liu, K. Kou, J. Morais, and P. Dang, "Signal moments for the shorttime Fourier transform associated with Hardy-Sobolev derivatives," Mathematical Methods in the Applied Sciences, vol. 38, no. 13, pp. 2719-2730, Sept 2015.

[29] M. Riera-Guasp, M. Pineda-Sanchez, J. Perez-Cruz, R. Puche-Panadero, J. Roger-Folch, and J. Antonino-Daviu, "Diagnosis of Induction Motor Faults via Gabor Analysis of the Current in Transient Regime," IEEE Trans. Instrum. Meas., vol. 61, no. 6, pp. 1583-1596, June 2012.

Jordi Burriel-Valencia received his Ms.C. degree in Informatics Engineering from the Universitat Politècnica de València, Spain, in 2010. Currently he is a Ph.D. student, working in the Department of Electrical Engineering of the Universitat Politècnica de València, and focuses his research on the development of expert systems for fault diagnosis.

Ruben Puche-Panadero (M09) received his M.Sc. degree in Automatic and Electronic Engineering in 2003, and its Ph.D. degree in Electrical Engineering in 2008, both from the Universitat Politècnica de València. He joined the Universitat Politècnica de València in 2006 and he is currently Associate Professor of Control of Electrical Machines. His research interests focus on induction motor diagnosis, numerical modelling of electrical machines, and advanced automation processes and electrical installations.

Javier Martinez-Roman received his Ph.D. degree in Electrical Engineering from the Universitat Politècnica de València, Spain, in 2002. Currently he is Associate Professor with the Universitat Politècnica de València. His research interests are electrical machines and drives. He has participated in various international projects supported by the European Union. He has published several papers on electrical machines in international journals and conference proceedings.

Angel Sapena-Bano obtained its M.Sc. degree in 2009 and its Ph.D. degree in 2014 from the Universitat Politècnica de València (Spain), both in Electrical Engineering. Currently he works as a researcher in the Institute for Energy Engineering of Universitat Politècnica de València. His research interests focus on induction motor diagnostics, numerical modelling of electrical machines, and advanced automation processes and electrical installations.

Manuel Pineda-Sanchez (M02) received his M.Sc. degree in 1985 and his Ph.D. degree in 2004 from the Universitat Politècnica de València, both in Electrical Engineering. Currently he is an Associate Professor in the Department of Electrical Engineering of the Universitat Politècnica de València. His research interests include electrical machines and drives, induction motor diagnostics and numerical simulation of electromagnetic devices. 